\title{
Deficiency of 53BP1 inhibits the radiosensitivity of colorectal cancer
}

\author{
YONG XIAO*, XIUMEI ZHENG* , AI HUANG, TAO LIU, TAO ZHANG and HONG MA \\ Cancer Center, Union Hospital, Tongji Medical College, Huazhong University \\ of Science and Technology, Wuhan, Hubei 430022, P.R. China
}

Received May 26, 2016; Accepted July 11, 2016

DOI: $10.3892 /$ ijo.2016.3629

\begin{abstract}
The present study aimed to observe the influence of p53 binding protein 1 (53BP1) silencing on the radiosensitivity of colorectal cancer (CRC) cells and investigated the potential underlying mechanisms. The differences in radiosensitivity among four CRC cells were detected by the clone formation assay, while the expression of their 53BP1 was detected by the western blot analysis. HCT116 cells with relatively high expression of 53BP1 were selected to silence the expression of 53BP1 by shRNA intervention. The influence on proliferation, apoptosis, and cell cycle distribution was detected by immunofluorescent staining of Ki-67 and flow cytometry. The expression of relevant proteins in the apoptotic pathway ATM-CHK2-p53 was further analyzed by western blot analysis. The expression of 53BP1 was found to be closely related to the radiosensitivity of the CRC cells. Decreased expression of 53BP1 led to the tolerance of HCT116 cells to radiation. The detection of tumor proliferation, apoptosis, and cell cycle showed that decreased expression of 53BP1 resulted in an increased S-phase percentage of HCT116 cells, an increased proliferating rate, and a decreased apoptotic rate after radiation. The analysis of the molecular pathway showed that the reduced expression of 53BP1 decreased the protein expression of ATM, CHK2, and the phosphorylated products associated with the p53 apoptotic pathway. In conclusion, decreased
\end{abstract}

Correspondence to: Dr Hong Ma or Dr Tao Zhang, Cancer Center, Union Hospital, Tongji Medical College, Huazhong University of Science and Technology, 1277 Jiefang Avenue, Wuhan, Hubei 430022, P.R. China

E-mail: wudajianzhu2004@163.com

E-mail: zhangtao_xiaohua@126.com

${ }^{*}$ Contributed equally

Abbreviations: 53BP1, p53 binding protein 1; CRC, radiosensitivity of colorectal cancer; DSBs, double-strand breaks; DDR, damage repair; ATM, ataxia-telangiectasia mutated kinase; CHK2, checkpoint kinase-2; RT-PCR, reverse transcription-polymerase chain reaction; SER, sensitization enhancing ratio

Key words: 53BP1, ATM-CHK2-p53 pathway, colorectal cancer, DNA damage repair, radiosensitivity expression of 53BP1 leads to radiotolerance of CRC cells, and the underlying mechanism is probably related to the decreased expression of relevant proteins in the ATM-CHK2-p53 pathway, which affects cell cycle, apoptosis and proliferation.

\section{Introduction}

Radiotherapy, following surgery and chemotherapy, is one of the most important therapies for colorectal cancer (CRC) in the advanced stage. Multiple clinical studies have verified that radiotherapy can, for example, lower preoperative staging and improve the operative resection rate and progressionfree survival of patients (1). However, due to the indigenous biological properties of CRC, the tumor tissue itself shows low radiosensitivity. Moreover, radiotolerance is limited during the radiotherapy for CRC because of many normal risks to organs (such as small intestine and kidney) in its anatomical environment. Although radiotherapy instruments and technologies are constantly being upgraded, they exhibit restricted capacity of simultaneously increasing the dosage at the tumor region and decreasing the exposure to normal organs, thus limiting their application in gastrointestinal tumors (2). Therefore, enhancing the radiosensitivity of CRC cells based on the indigenous biological properties of tumors has become a critically urgent challenge to be addressed.

Cellular responses to external damage (due to radiotherapy and chemotherapy) depend on the self-repairing ability of cells, such as the ability to repair DNA double-strand breaks (DSBs) (3). Multiple signaling pathways are involved in the process of DSB repair, including homologous recombination repair, nonhomologous end joining, cell cycle regulation and apoptotic pathways $(4,5)$. p53 binding protein 1 (53BP1) has been confirmed to contribute to apoptosis induction in DNA damage repair (DDR) and helps in determining the cellular response to different therapies $(6,7)$. 53BP1 was originally believed to be the binding protein of tumor suppressor gene $\mathrm{p} 53$ and thought to be involved in p53-mediated apoptosis. 53BP1 has an important function as a significant reaction medium at the early stage in signal transduction pathways following DNA damage (6). Also, tumor tissues differ in their degree of 53BP1 deletion, which is closely related to the malignant staging of tumor cells and histological staging, and affects the sensitivity to drug, which is manifested as resistance to treatment $(7,8)$. Moreover, 53BP1 can affect tumor (breast 
cancer and glioma) responses to radiotherapy $(9,10)$, although it has been rarely reported how abnormal expression of 53BP1 disturbs the response of CRC to radiotherapy. A previous study showed that epidermal growth factor receptor-tyrosine kinase inhibitor-icotinib hydrochloride enhanced the sensitivity of CRC to radiotherapy by increasing the expression of 53BP1 (11). In contrast, the radiosensitization effect of icotinib hydrochloride was notably eliminated when the expression of 53BP1 of CRC cells was decreased by shRNA, which implied that the abnormal expression of 53BP1 could influence the radiosensitivity of CRC. However, the exact effects and potential underlying mechanisms remain to be resolved.

Recent studies showed that on external damage, cells may activate multiple signaling pathways, which may contribute to cellular injury repair and induce apoptosis. The ataxiatelangiectasia mutated kinase (ATM)-checkpoint kinase-2 (CHK2)-p53 pathway plays a pivotal role in the DDR process. When activated, it may induce cell cycle arrest, leading to the activation of p53-related apoptotic pathways, which trigger apoptosis $(12,13) .53 \mathrm{BP} 1$ is closely related to the ATM-CHK2-p53 pathway because of its special structure. It possesses two Tudor domains, which promote it to interact with ATM to involve in DDR $(8,14,15)$, and a C-terminal BRTC domain, which activates CHK1 and CHK2 of the cell cycle to regulate the G1/S, S and G2/M checkpoints, phosphorylate $\mathrm{p} 53$, and then induce p53-dependent apoptosis $(15,16)$. Thus, considering the mediator-featured structure of 53BP1, we speculated that the abnormal expression of 53BP1 could affect the tumor cell proliferation, apoptosis, and cell cycle distribution through interfering the ATM-CHK2-p53 pathway.

Previous studies have found that the expression of 53BP1 was deficient in CRC tissue, which was an early factor of poor prognosis. Therefore, we hypothesized that this deficiency may be an intrinsic factor that influences the response of colorectal tumors to radiotherapy. The present study aimed to verify the correlation between the expression of 53BP1 and radiosensitivity in various CRC cell lines and to investigate how 53BP1 affects the response to radiotherapy by disturbing the expression of 53BP1 in CRC cells in vitro. Moreover, the expression of proteins related to the ATM-CHK2-p53 pathway was also detected to explore the intrinsic factors of 53BP1 affecting the radiotherapy efficacy mechanically.

\section{Materials and methods}

Cell culture and animal care. HCT116, SW620, Caco2 and LoVo cells were cultured in an RPMI-1640 medium containing $10 \%$ fetal bovine serum (FBS) at $37^{\circ} \mathrm{C}, 5 \% \mathrm{CO}_{2}$, and saturated humidity. Passages were performed to maintain monolayer growth. Cells were collected at the exponential growth phase for subsequent experiments. Female athymic nude mice (nu/nu; body weight, 20-25 g; 6-10 weeks of age) were purchased from Beijing HFK Bioscience Co., Ltd. (Beijing, China). The care and treatment of all experimental mice was in accordance with the institutional guidelines.

Cell transfection. This study used GCACAAGAACTTATGG AAAGT as the shRNA sequence of 53BP1 and TTCTCCGA ACGTGTCACGT as the shRNA sequence of the control group. The lentivirus plasmids containing the shRNA and green fluorescent protein lentivirus vector containing 53BP1 shRNA were purchased from Shanghai Genechem Co., Ltd. (Shanghai, China). The concentrated virus solution and HCT116 cells were co-cultured, and their fluorescence was observed by optical microscopy to confirm successful transfection. The transfection rates were verified by western blot analysis and reverse transcription-polymerase chain reaction (RT-PCR).

Clone formation assay. The cells at the exponential growth phase were digested into single-cell suspensions. After counting, the living cells were inoculated onto a 6-well plate at 100-5,000 cells/well. When cells grew in an adherent manner after a 24-h incubation, they were exposed to different radiation dosages $(0,2,4,6,8$ and $10 \mathrm{~Gy})$ with a dosage rate of $2 \mathrm{~Gy} / \mathrm{min}$. Incubation was terminated after 10-14 days when $>50$ cells formed a clone in a visible cell mass. The cells were fixed with methanol and stained with Giemsa; colonies containing at least 50 cells were counted, and cell survival curves were plotted using the multitarget click model to compare the difference of radiosensitivity. Relative parameters, such as mean lethal dose $\left(D_{0}\right)$, quasi-threshold dose $\left(D_{\mathrm{q}}\right)$, extrapolation number $(N)$, surviving fraction at $2 \mathrm{~Gy}\left(\mathrm{SF}_{2}\right)$, and a ratio of $\mathrm{SF}_{2}$, termed as sensitization enhancing ratio (SER), were calculated. Three parallel tests were set.

Evaluation of tumor proliferation by immunofluorescent staining of $\mathrm{Ki}-67$. The proliferation antigen $\mathrm{Ki}-67$ was used to assess the proliferation rate of tumor. At the exponential growth phase, $2 \times 10^{5}$ cells $/ \mathrm{ml}$ were plated in chamber slides. After $24 \mathrm{~h}$ of irradiation (at 0,2 and $4 \mathrm{~Gy}$ ), the cells were fixed and permeabilized with $0.2 \%$ Triton X-100 (Wuhan Boster Biological Technology, Ltd., Wuhan, China). They were then incubated with anti-Ki-67 antibody (1:50, 19972-1-AP; Proteintech Group, Rosemont, IL, USA), and then with Alexa Fluor 488-conjugated goat anti-rabbit (Proteintech Group) at a dilution of 1:200. Finally, the sections were counterstained with 6-diamidino-2-phenylindole dihydrochloride (Vector Laboratories, Inc., Burlingame, CA, USA). The sections were examined on a confocal laser scanning microscope (Olympus, Tokyo, Japan) equipped with a camera. To determine the percentage of positive cells with $\mathrm{Ki}-67$, at least 500-1,000 tumor cells per slide were counted, and the number of $\mathrm{Ki}-67-$ positive cells was scored and the positive rate was counted.

Cell cycle detection by flow cytometry. A total of $2 \times 10^{5}$ cells $/ \mathrm{ml}$ at the exponential growth phase were collected and inoculated onto a 6-well plate. When the cells attached to the wall after a 24-h incubation, they were exposed to different irradiation doses (at 0,2 and 4 Gy). The cells were then digested with trypsin $24 \mathrm{~h}$ after irradiation. After overnight fixation with $70 \%$ ethanol, the cells were digested again with $1 \%$ RNase for $30 \mathrm{~min}$ at $37^{\circ} \mathrm{C}$, followed by $30-\mathrm{min}$ staining with $20 \mathrm{mg} / \mathrm{ml}$ propidium iodide (PI). Finally, the cell cycle was detected using a flow cytometer (BD Biosciences, San Jose, CA, USA).

Apoptosis detection by flow cytometry. The cells were routinely digested, washed, suspended gently with $500 \mu 1$ combining solution, gently blended with $5 \mu$ 1 Annexin V-FITC, and finally 
blended with $5 \mu 1$ PI using an Annexin V-FITC apoptosis detection kit, according to the manufacturer's instructions. Then, after 10 -min incubation at room temperature $\left(20-25^{\circ} \mathrm{C}\right)$ in darkness, cells were analyzed using a flow cytometer (BD Biosciences). Ten thousand cells in each sample were analyzed, and the CellQuest software (BD Biosciences) was employed to analyze the data.

Western blot analysis. The cells were lysed in a radio immunoprecipitation assay lysis buffer for $30 \mathrm{~min}$ at $4^{\circ} \mathrm{C}$ and centrifuged at $12,000 \mathrm{x}$ g for $5 \mathrm{~min}$. The supernatant was collected, combined with sodium dodecyl sulfate (SDS) buffer, and heated to $100^{\circ} \mathrm{C}$ for $5 \mathrm{~min}$. The proteins were separated by $10 \%$ SDS-PAGE and blotted to the polyvinylidene fluoride membranes, which were incubated with primary antibodies against 53BP1 (ab175933, 1:2,000; Abcam, Cambridge, MA, USA), ATM (\#21147, 1:1,000; Signalway), ATMpS1981 (5883, 1:1,000; Cell Signaling Technology, Danvers, MA, USA), CHK2 (AP4999a, 1:1,000; Abgent, San Diego, CA, USA), CHK2pT68 (2197, 1:1,000; Cell Signaling Technology), p53 (AM2244B, 1:1,000; Abgent), p-P53 (9286, 1:1,000; Cell Signaling Technology), caspase-9 (10380-1-AP, 1:1,000; Proteintech Group), caspase-3 (BS1518, 1:1,000; Bioworl, Dublin, OH, USA), Bax (1063, 1:1,000; EpiGentek, Farmingdale, NY, USA), Bcl-2 (2870-P, 1:1,000; Cell Signaling Technology), or $\beta$-actin antibody (1:1,000; Santa Cruz Biotechnology, Santa Cruz, CA, USA) at $4^{\circ} \mathrm{C}$ overnight. The membranes were washed with Tris-buffered saline and Tween-20 three times, incubated with secondary antibodies for $2 \mathrm{~h}$ at room temperature, and visualized by enhanced chemiluminescence. Band intensities were analyzed by the Gel-Pro analysis program.

Quantitative RT-PCR. The total RNA was extracted with a TRIzol reagent. Then, an RT-PCR kit (Thermo Fisher Scientific, Inc., Waltham, MA, USA) was used to reversely transcribe RNA into cDNA. StepOne and StepOnePlus RealTime PCR Systems (Applied Biosystems) were adopted for amplification analysis. More than three parallel tests were set for all trials.

Establishing the tumor-bearing nude mouse model. Cultured tumor cells were collected to prepare tumor cell suspensions with an RPMI-1640 (1:1) at a concentration of approximately $2 \times 10^{7}$ cells $/ \mathrm{ml}$ for the following animal experiments. Tumor cells $(0.1 \mathrm{ml})$ (HCT116 and HCT116 with 53BP1 silencing) were subcutaneously injected into the posterior limbs of the mice and observed for 5-7 days. When tumors reached a diameter of $10 \mathrm{~mm}$, all nude mice were randomly divided into different groups $(n=3)$ to receive different treatments as observation by radiotherapy. For the radiotherapy group, irradiation was performed using a 6 MV linear accelerator (MDX, Siemens) with an exposure field of $5 \times 5 \mathrm{~cm}^{2}$, and the total dose was $10 \mathrm{~Gy}(2 \mathrm{~Gy} / \mathrm{day}$ for 5 days). Since the beginning of the treatment, the caliper was used to measure the longest diameter $\mathrm{L}$ and the shortest diameter $\mathrm{W}$ of each tumor every other day, and the tumor volume was calculated based on the following formula: Tumor volume $=\left(\mathrm{Lx} \mathrm{W}^{2}\right) / 2 \mathrm{~mm}^{3}$. The growth time and volume of each tumor were recorded.
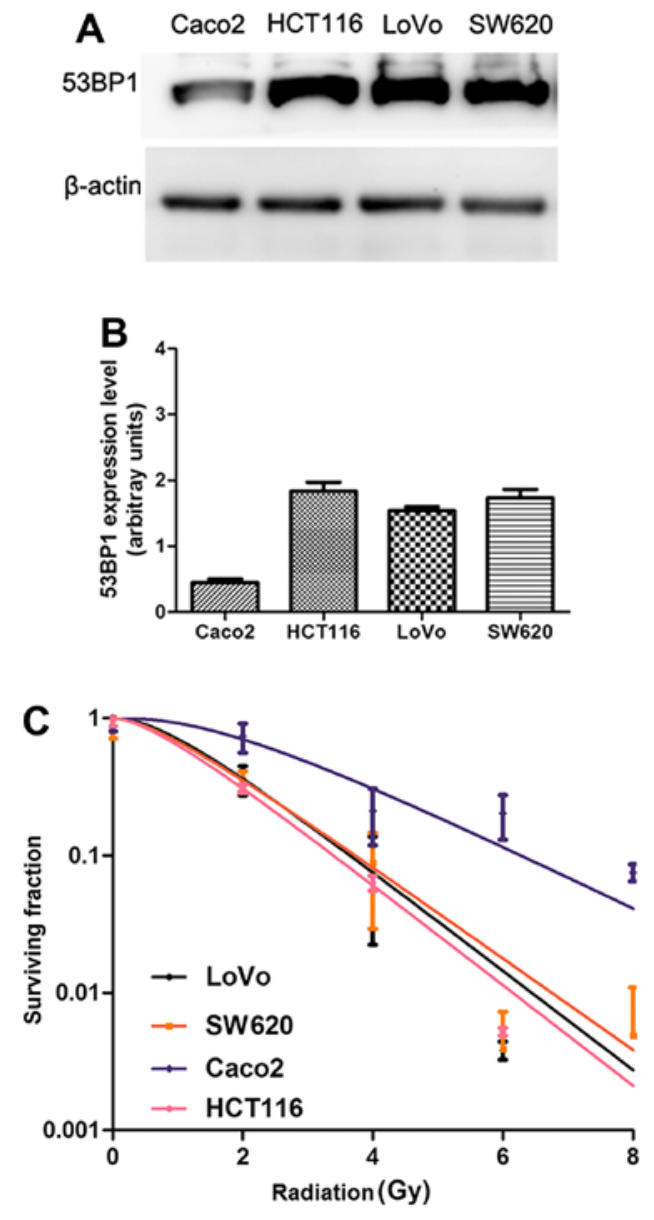

Figure 1. Expression of 53BP1 is related to radiation sensitivity in the four CRC lines. (A) 53BP1 protein level in CRC lines was detected using western blot analysis. (B) Histograms of quantification expression of 53BP1 protein based on the mean of three independent experiments; $\beta$-actin was used as the endogenous control. Error bar shows the standard error. (C) Survival curve of various CRC cell lines. The radiosensitivity of various CRC cell lines was detected by the clonogenic assay. The multitarget click model was used to fit the experimental data. Error bars show the standard error of the mean for three independent experiments.

Statistical analysis. SPSS 13.0 software was used for the statistical analysis. Quantitative data were expressed as mean 13.0. Mean value comparison among multiple groups was performed by one-way analysis of variance, and the comparison between the two groups was carried out by applying the Q-test. $\mathrm{P} \leq 0.05$ indicated statistical significance.

\section{Results}

Expression of 53BP1 is closely related to the radiosensitivity of CRC cells. To investigate the correlation between the expression of 53BP1 and radiosensitivity of CRC cells, the expression of 53BP1 in the four CRC cell lines (HCT116, SW620, Caco2 and LoVo cells) was detected in vitro by the western blot analysis (Fig. 1A and B), and the radiosensitivity of these intestinal cancer cell lines was simultaneously detected by the clone formation assay (Fig. 1C). The results showed that the expression of 53BP1 was closely related to the radiosensitivity of CRC cells. The HCT116 cell line with the high expression of 53BP1 was relatively sensitive to irradiation with lower values of $\mathrm{SF}_{2}, D_{0}, D_{\mathrm{q}}$, and $N$ in the cell survival curve analysis, while 
Table I. The relative parameters of cell survival curves after irradiation.

\begin{tabular}{lllll}
\hline Parameter/cell lines & Caco2 & LoVo & SW620 & HCT116 \\
\hline$D_{0}$ & 1.90 & 1.20 & 1.30 & 1.18 \\
$D_{\mathrm{q}}$ & 3.44 & 1.37 & 1.03 & 0.93 \\
$N$ & 2.812 & 2.137 & 1.792 & 1.786 \\
$\mathrm{SF}_{2}$ & 0.74 & 0.36 & 0.35 & 0.31 \\
\hline
\end{tabular}

$D_{0}$, lethal dose; $D_{\mathrm{q}}$, quasi-threshold dose; $N$, extrapolation number; $\mathrm{SF}_{2}$, surviving fraction at $2 \mathrm{~Gy}$.

Table II. Effect of 53BP1 silencing on radiosensitivity in HCT116 cells.

\begin{tabular}{lcc}
\hline Parameter/cell lines & Control & 53BP1 silence \\
\hline$D_{0}$ & 0.98 & 1.28 \\
$D_{\mathrm{q}}$ & 1.50 & 2.35 \\
$N$ & 1.53 & 1.84 \\
$\mathrm{SF}_{2}$ & 0.30 & 0.49 \\
$\mathrm{SER}$ & & 0.77 \\
\hline
\end{tabular}

$D_{0}$, lethal dose; $D q$, quasi-threshold dose; $N$, extrapolation number; $\mathrm{SF}_{2}$, surviving fraction at $2 \mathrm{~Gy}$; SER, sensitization enhancing ratio.

the Caco2 cell line with the low expression of 53BP1 was relatively tolerant to irradiation with higher values of $\mathrm{SF}_{2}, D_{0}, D_{\mathrm{q}}$, and $N$ in the cell survival curve analysis (Table I).

Decreased expression of 53BPI inhibits the radiosensitivity of HCT116 cell line. The HCT116 cell line with relatively high expression of 53BP1 was selected, and stable HCT116 cell stains were constructed by RNA-intervened lentiviral vector transfection by decreasing the expression of 53BP1 via shRNA. The effective transfection rate was verified by western blot analysis and RT-PCR (Fig. 2A and B). Then, the effect of transfection on the cell radiosensitivity was detected, which showed that the decrease in the expression of 53BP1 increased $\mathrm{SF}_{2}, D_{0}, D_{\mathrm{q}}$, and $N$ values, and decreased the value of SER. It indicated that the decreased expression of 53BP1 obviously inhibited the radiosensitivity of the HCT116 cells (Table II and Fig. 2C).

Decreased expression of 53BP1 increases the proliferation rate of HCT116 cells after irradiation. To determine the association between the radiosensitivity of HCT116 cells after 53BP1 silencing and tumor proliferation, the expression of $\mathrm{Ki}-67$ was investigated by immunofluorescent staining. As shown in Fig. 3, the results revealed that the decrease in the expression of 53BP1 obviously increased the expression of Ki-67 compared with the control group. The proliferation rate was $31 \pm 4$ vs. $18 \pm 4 \%$ before irradiation $(\mathrm{P}<0.05)$. After irradiation of 2 and $4 \mathrm{~Gy}$, the 53BP1-silenced group still revealed stronger capacity to induce tumor proliferation compared with the control
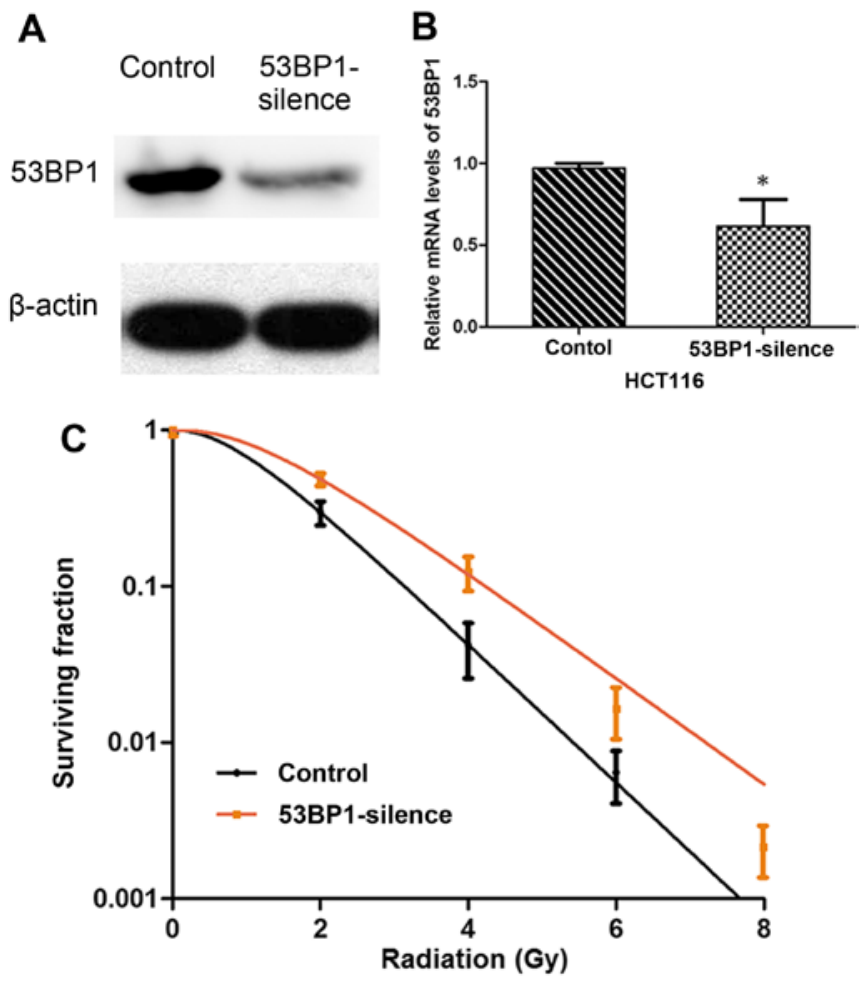

Figure 2. Decrease in the expression of 53BP1 inhibits the radiation sensitivity of HCT116 cells. (A and B) Western blot analysis and RT-PCR were used to confirm the transfection efficiency. (C) Survival curve of control and 53BP1 silencing cells of HCT116. The radiosensitivity of control and 53BP1 silencing cells of HCT116 was detected by the clonogenic assay.
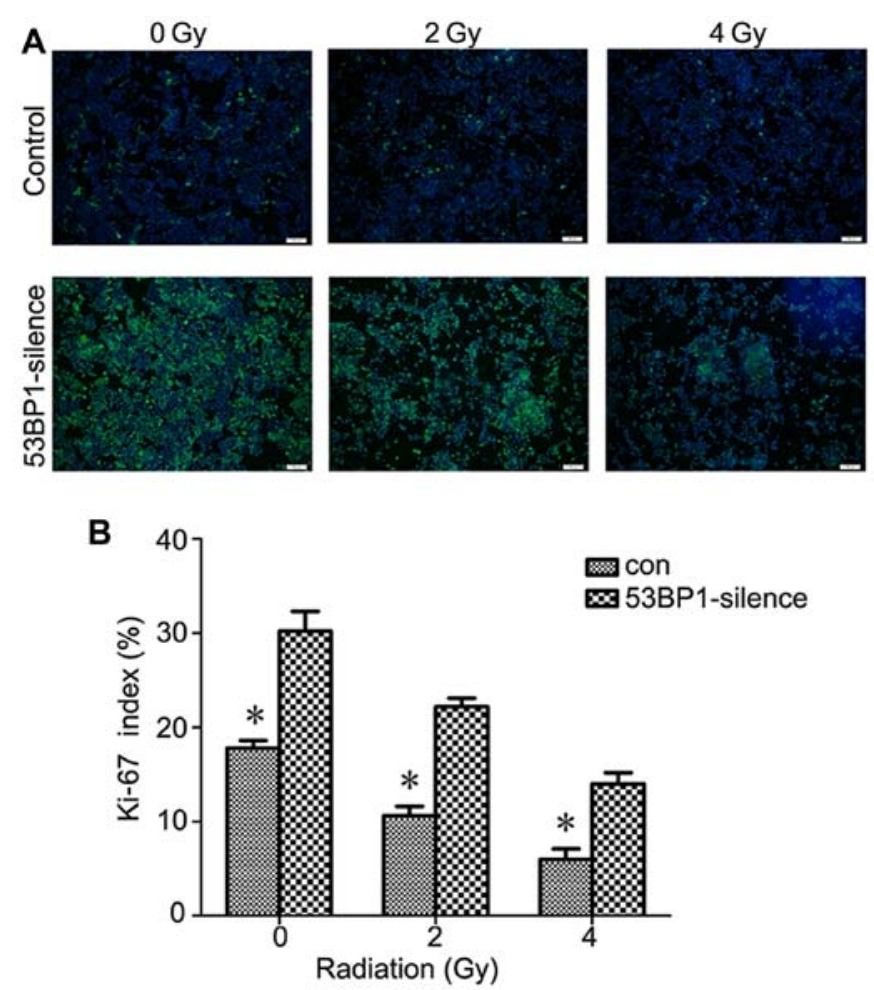

Figure 3. Decrease in the expression of 53BP1 increased Ki-67 proliferative marker in HCT116 cells. (A) Immunofluorescence staining of Ki-67 in HCT116 cells after the irradiation of 0,2 and 4 Gy (magnification, x100). (B) Average proliferative index was determined by counting positive cells per microscopic field, and histograms of quantification of control and 53BP1 silencing cells of HCT116 was plotted. Columns, mean; error bars, standard error of mean from three independent experiments. ${ }^{*} \mathrm{P}<0.05$. 

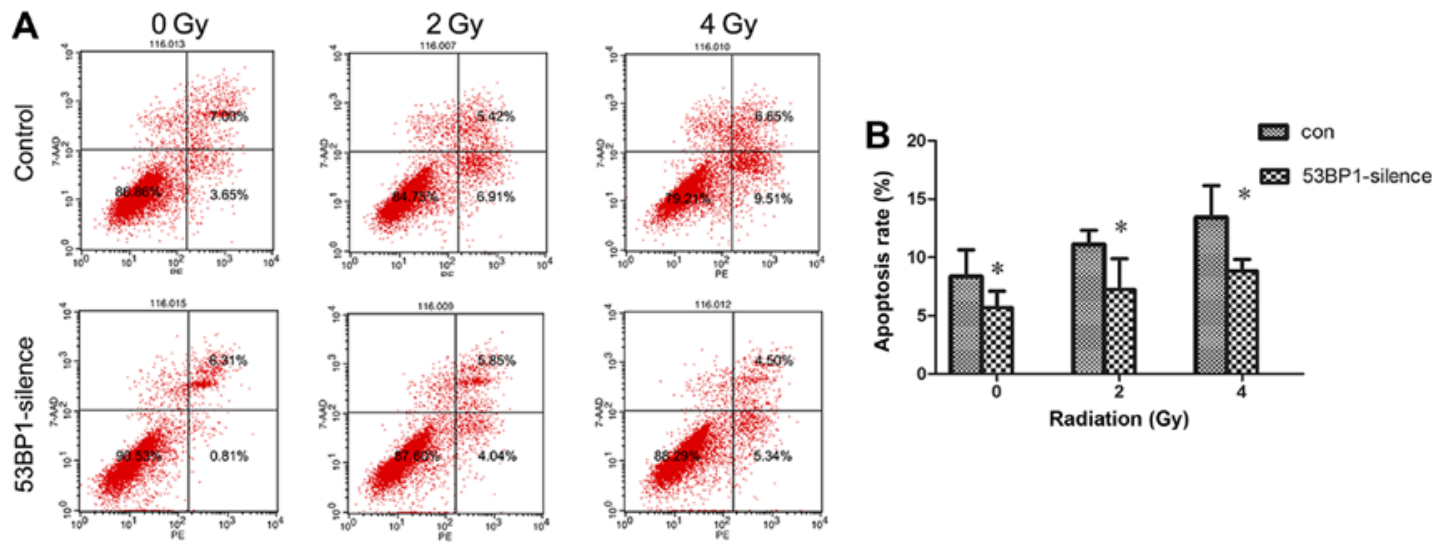

Figure 4. Decrease in the expression of 53BP1 decreased HCT116 cell apoptosis after radiation. (A) Apoptosis assays by flow cytometry in HCT116 after radiation. The doses are 0,2 and $4 \mathrm{~Gy}$. The apoptotic rate was calculated as the percentage of Annexin V positive cells. (B) Histograms of quantification of apoptosis rates of control and 53BP1 silencing cells of HCT116. Columns, mean; error bars, standard error of mean from three independent experiments. "P<0.05.
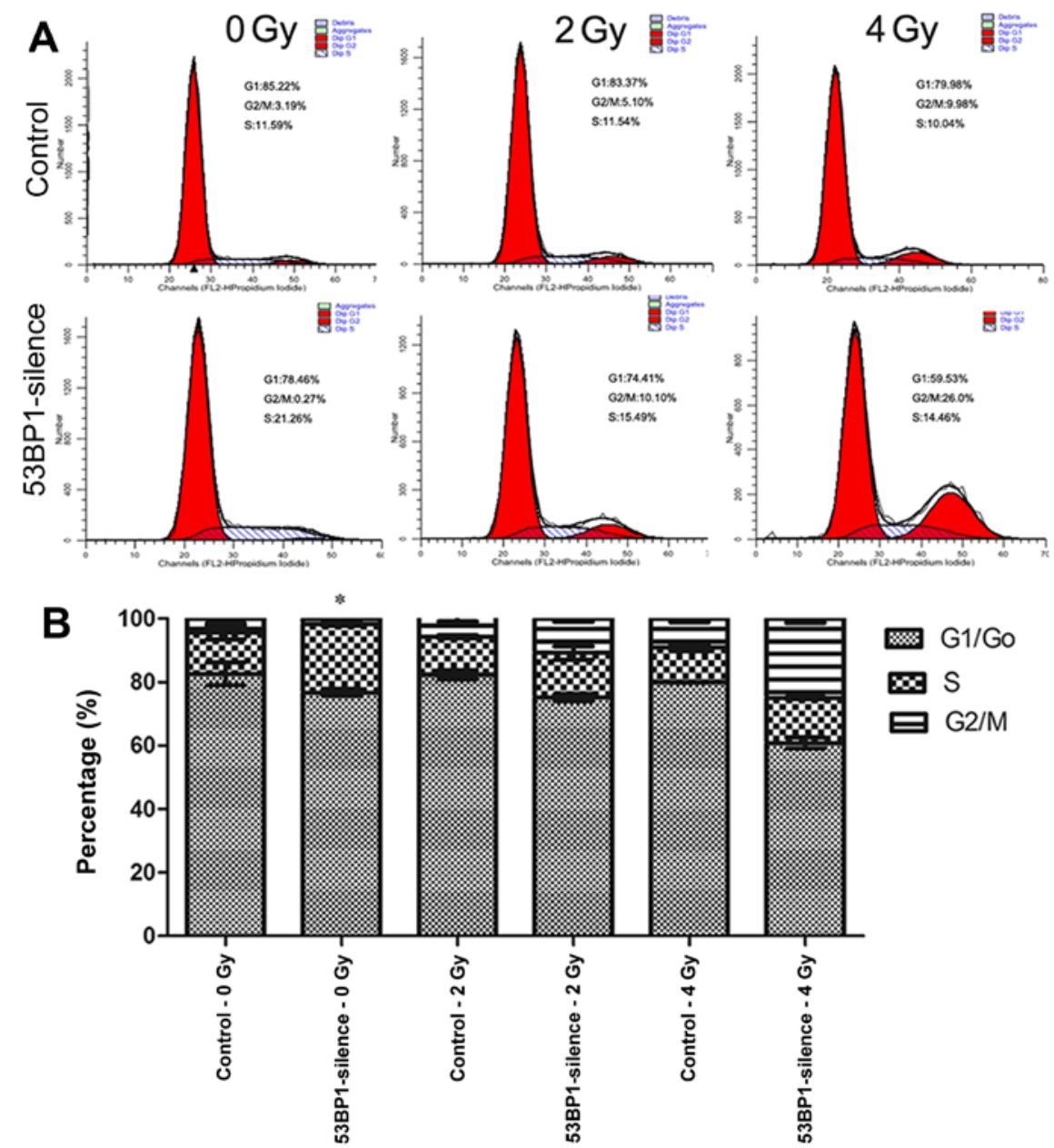

Figure 5. 53BP1 silencing increases S-phase accumulation in HCT116 and HT29 cells. (A) Cell cycle distribution in HCT116 control and 53BP1-silenced cells after radiation. The doses are 0,2 and $4 \mathrm{~Gy}$. (B) Histogram plots of HCT116 cell cycle distribution. Columns, mean; error bars, standard error of mean from three independent experiments. " $\mathrm{P}<0.05$.

group. The proliferation rate was $22 \pm 3$ vs. $11 \pm 4 \%$ for $2 \mathrm{~Gy}$ and $14 \pm 5$ vs. $6 \pm 2 \%$ for $4 \mathrm{~Gy}$. The difference between the two groups was obvious $(\mathrm{P}<0.05)$.

Decreased expression of 53BP1 reduces the apoptotic rate of HCT116 cells after radiotherapy. The effect of the decreased expression of 53BP1 on tumor cell apoptosis was investigated after radiotherapy. The apoptotic rate of tumor cells before and after intervention at $24 \mathrm{~h}$ after exposure to irradiation doses of 0,2 and $4 \mathrm{~Gy}$ was detected using flow cytometry (Fig. 4A). The results showed that the decrease in the expression of 53BP1 significantly reduced apoptosis of HCT116 cells $(8.49 \pm 1.88$ vs. 
A

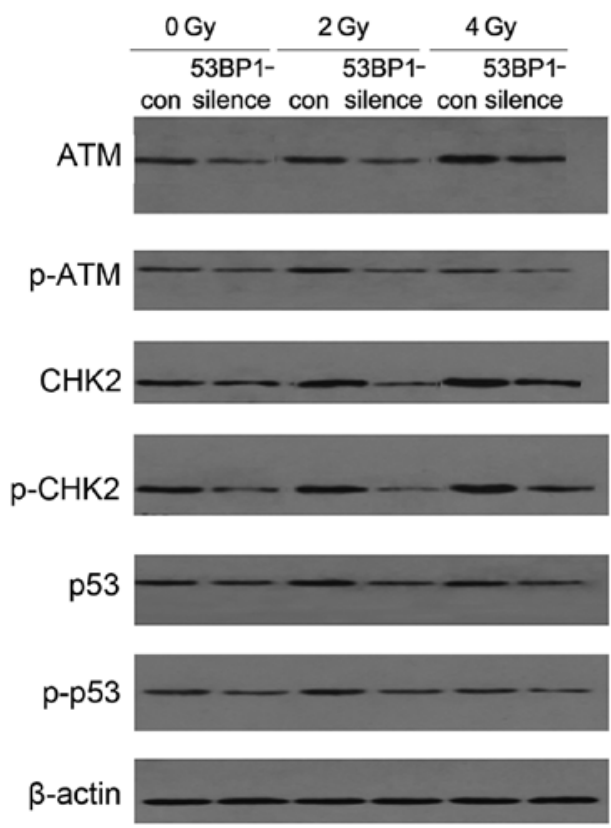

C

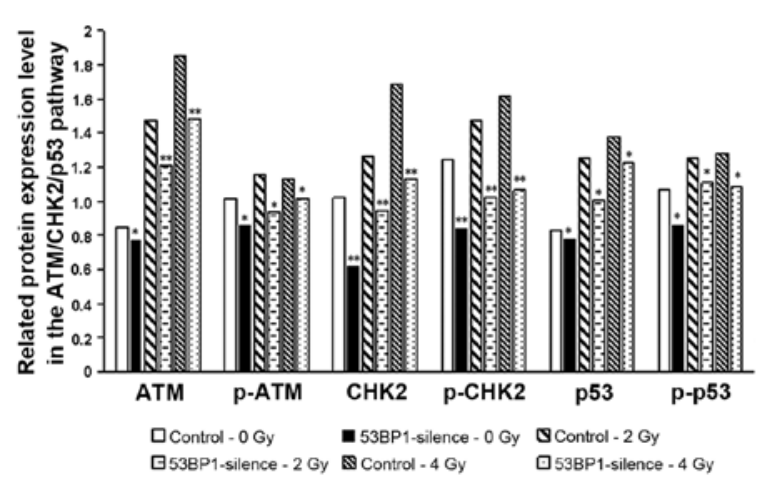

B

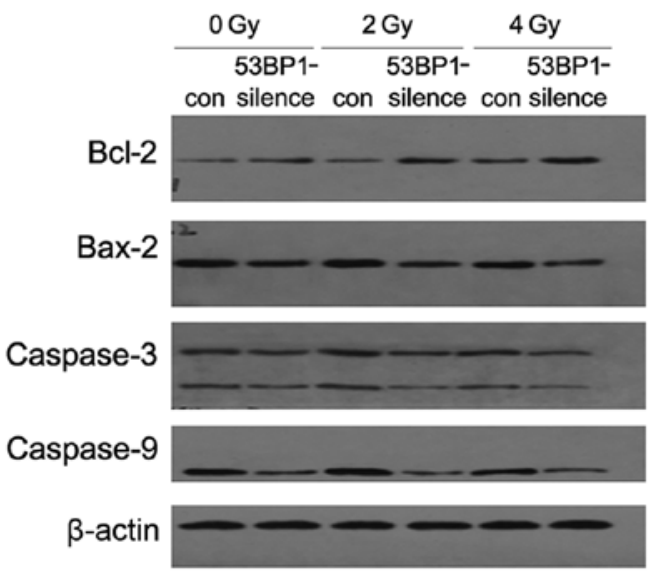

D

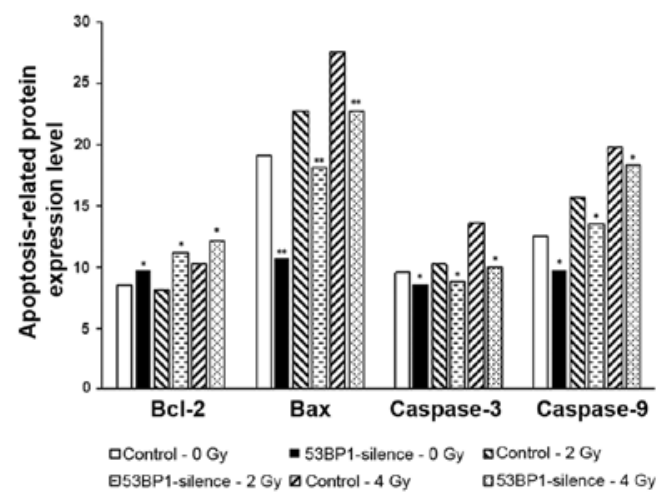

Figure 6. 53BP1 silencing inhibits related protein expression in the ATM-CHK2-p53 pathway. (A) Western blot detection of proteins and their phosphorylated variants in the ATM-CHK2-p53 pathway in HCT116 with 53BP1-silenced and control cells after radiation. The doses are 0, 2 and 4 Gy. (B) Western blot detection of apoptosis-related protein caspase-9, caspase-3, Bax and anti-apoptotic protein Bcl-2 in HCT116 with 53BP1-silenced and control cells after radiation. The doses are 0, 2 and 4 Gy. (C and D) Quantitative detection of ATM-CHK2-p53 and apoptosis-related protein bar chart. Columns, means; error bars, standard error of mean from three independent experiments. " $\mathrm{P}<0.05$.

$4.19 \pm 1.08 \% ; \mathrm{P}<0.05)$; the decrease in the expression of 53BP1 reduced apoptosis after irradiation $(10.58 \pm 1.16$ vs. $5.91 \pm 2.65 \%$ for $2 \mathrm{~Gy}, 13.43 \pm 3.15$ vs. $8.82 \pm 1.17 \%$ for $4 \mathrm{~Gy}$; $\mathrm{P}<0.05)($ Fig. $4 \mathrm{~B})$

Decreased expression of 53BP1 increases the percentage of S-phase of HCT116 cells after irradiation. The effect of the decreased expression of 53BP1 on the cell cycle of HCT116 cells after irradiation was investigated using the flow cytometer (Fig. 5A). The results showed that the decrease in the expression of 53BP1 remarkably increased the percentage of the $\mathrm{S}$-phase in tumor cells $(21.78 \pm 0.73$ vs. $13.09 \pm 2.12 \% ; \mathrm{P}<0.05)$, indicating that the decreased expression of 53BP1 ushered tumor cells into the proliferation cycle with an accelerated proliferation rate. This result was consistent with the results of previous studies. After irradiation with an exposure to doses 2 and $4 \mathrm{~Gy}$, the two 53BP1 silencing groups exhibited relatively higher percentages of $\mathrm{S}$-phase compared with the control groups $(14.92 \pm 0.71$ vs. $11.91 \pm 0.53 \%$ for $2 \mathrm{~Gy}, 14.23 \pm 0.32$ vs $10.80 \pm 1.07 \%$ for $4 \mathrm{~Gy}$ ) although the difference between these two exposure groups was not significant (Fig. 5B). This indicates that the decrease in the expression of 53BP1 ushers tumor cells into a high-proliferation status, which inhibits the efficacy of regular radiotherapy.

Decreased expression of 53BP1 inhibits the expression of relevant proteins in the ATM-CHK2-p53 pathway. The ATM-CHK2-p53 pathway plays a pivotal role in the DNA injury repair process and that activation of this pathway may induce the cell cycle arrest, aggravate the DNA injury, and trigger the expression of p53-related apoptotic proteins. Therefore, western blot analysis was used to detect the expression of relevant proteins and their phosphorylated products in the ATM-CHK2-p53 pathway; apoptosis-related proteins caspase-9, caspase-3 and Bax; and the anti-apoptosis protein Bcl-2 before and after the decreased expression of 53BP1 and with or without radiation of 2 and $4 \mathrm{~Gy}$. The results showed that the decrease in the expression of 53BP1 decreased the expression of apoptotic pathway-related proteins ATM, CHK2 and p53 as well as their phosphorylated products (Fig. 6A and C); 

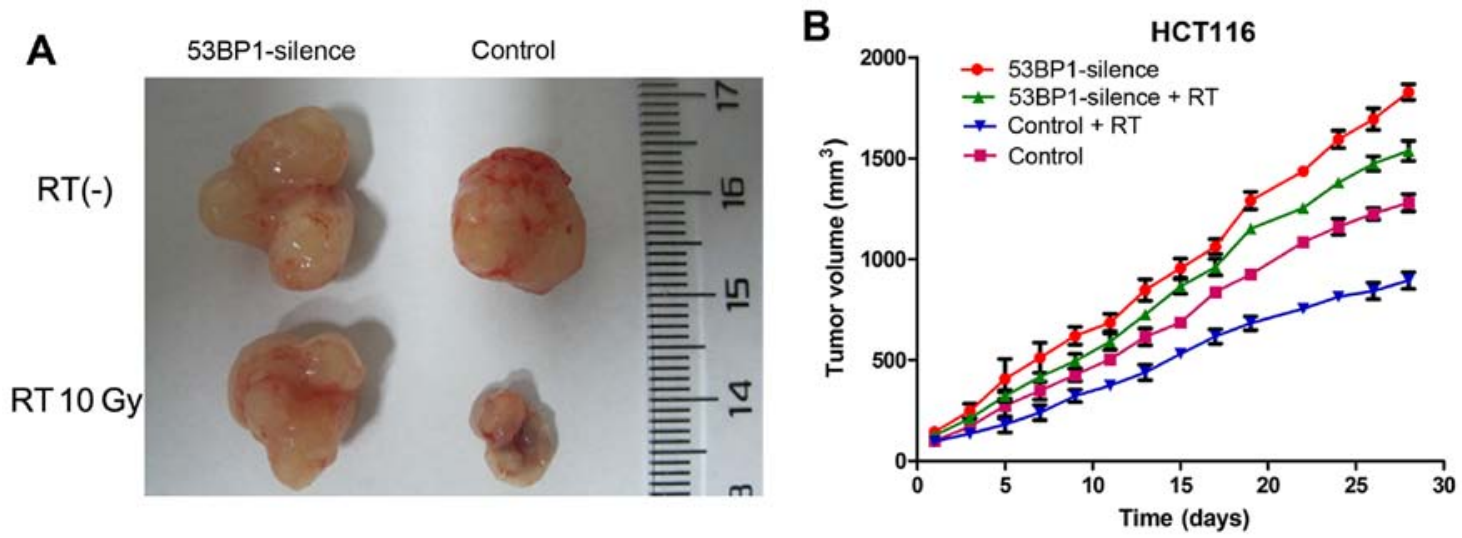

Figure 7. 53BP1 silencing decreases the radiation sensitivity in colorectal xenograft nude mice. The inhibition of in vivo tumor growth in nude mice treated with radiotherapy compared with the control group without radiation. (A) Representative tumor image of control and 53BP1-silenced HCT116 cells and with or without radiation. (B) Tumor growth curve of HCT116 with 53BP1-silenced and control cells with or without radiation. The dose is 10 Gy. Data points are the mean values of five animals in each group. Error bars are the standard error of mean of five experiments.

reduced the apoptosis-related proteins caspase- 9 , caspase-3 and Bax; and increased the expression of anti-apoptosis protein Bcl-2 (Fig. 6B and D), which may be the imminent cause of the decreased expression of 53BP1, resulting in an active proliferation of tumor cells. Similarly, the 53BP1-silenced group showed the reduced expression of the afore-mentioned apoptosis-related proteins and enhanced expression of the anti-apoptosis protein Bcl-2 compared with the control group after exposure to different radiation doses. This indicated that the decrease in the expression of 53BP1 led to the tolerance of tumor cells to irradiation via inhibiting the apoptosis-related ATM-CHK2-p53 pathway and the expression of relevant proapoptosis proteins and anti-apoptosis proteins.

Silencing 53BP1 in vivo induces the proliferation of tumor cells and inhibits reactivity to radiotherapy. To investigate the effect of 53BP1 on tumor proliferation and radiotherapy efficacy in vivo, the tumor-bearing nude mouse model was established. HCT116 and HCT116 with genetically silenced 53BP1 were subcutaneously injected into the right posterior limb of the mice. Tumor nodes were palpated after 7-10 days, and reached a diameter of $10 \mathrm{~mm}$ after 2 weeks. Subsequently, radiotherapy was performed $(10 \mathrm{~Gy} / 5 \mathrm{~F})$. The size of each tumor was recorded every other day starting from the beginning of treatment. When the tumors of a certain group reached a volume of $2 \mathrm{~cm}^{3}$, all nude mice were sacrificed to record the final tumor volume. The results showed that the tumors of the 53BP1-silenced group showed a relatively higher proliferation activity and reached a size of nearly $2 \mathrm{~cm}^{3}$ on the 28th day of the treatment. Then, all nude mice were sacrificed and the final volume of each tumor was recorded. The final tumor volumes of the 53BP1-silenced group without radiotherapy and the control group were $1.82 \pm 0.06$ and $1.28 \pm 0.06 \mathrm{~cm}^{3}$, respectively, with a significant difference $(\mathrm{P}<0.01)$. The 53BP1-silenced group with radiotherapy had a markedly higher proliferation velocity compared with the control group (final tumor volume: $1.54 \pm 0.07$ vs. $0.89 \pm 0.06 \mathrm{~cm}^{3} ; \mathrm{P}=0.01$ ), with a significant difference (Fig. 7). The results showed that the decrease in the expression of 53BP1 notably induced the growth of the transplanted colorectal tumor, which exhibited resistance to radiotherapy.

\section{Discussion}

Radiotherapy against CRC has now hit a bottleneck. Decades of radiosensitization studies have only realized a limited efficacy, which is primarily caused by the limited radiosensitivity of CRC cells. Therefore, the analysis of indigenous factors that affect tumor radiosensitivity is of crucial importance and has become an urgent problem that needs to be resolved. Multiple studies have shown that genetic stability is the necessary condition under which cells retain their normal function and maintain basic survival $(8,17)$. However, DNA is quite vulnerable to various factors, and this injury may lead to gene mutations, genomic instability, chromosomal loss and reorganization, apoptosis, or even cancerization if timely and precise repair is unavailable $(18,19)$. The DDR pathway plays an essential role in triggering DDR and maintaining the genomic stability of normal cells. Dysfunction of critical factors in the DDR pathway is closely related to the onset, development, and therapy resistance of multiple tumors (20-24). 53BP1 is a recently found cancer suppressor gene, which is also an important member of the DDR pathway family. It is involved in DDR, maintains genomic stability, and regulates apoptosis in coordination with $\mathrm{p} 53$ and ATM $(25,26)$. The deficiency of 53BP1 may lead to failed anchoring of broken chromosome ends, which lays the foundation for chromosomal aberration (22). Studies have shown that different degrees of 53BP1 deletion exist during the onset and development of multiple tumors and that this deletion is closely related to tumor staging, malignancy grading, and even therapy resistance $(8,17)$. Neboori et al (9) found that 53BP1 deletion resulted in an increased local recurrence rate of breast cancer after radiotherapy. Similarly, Han et al (27) found that the decrease in the expression of 53BP1 could enhance the response to DNA damaging agents. The deletion of the expression of 53BP1 in CRC tissue has been detected in previous studies, which was found to be related to the location of the tumors: the right half of the colon exhibited a higher 53BP1 deletion rate compared with the left half of the colon. Furthermore, 53BP1 deficiency was also an early risk factor of poor prognosis (28). Therefore, this study hypothesized that the deficiency of 53BP1 in CRC 
tissue probably played a part in influencing the response to radiotherapy.

The present study performed in vivo and in vitro trials to verify this hypothesis. The expression of 53BP1 in various CRC cell lines was detected by western blot analysis together with differences in their radiosensitivity. The results showed that the expression of 53BP1 was closely related to the radiosensitivity of CRC cells. HCT116 cells with the high expression of 53BP1 were relatively sensitive to radioactivity, while Caco2 cells with low expression of 53BP1 were relatively tolerant to radioactive rays. In this study, the expression of 53BP1 was further silenced via gene interference to investigate the effects of 53BP1 on the radiosensitivity of CRC. The HCT116 cell line with the high expression of 53BP1 was selected, and its stable cell line was constructed with an RNA-intervened lentiviral vector to decrease the expression of 53BP1 by shRNA. The effects of 53BP1 on radiosensitivity were detected by the clone formation assay before and after 53BP1 silencing. The results showed that the decrease in the expression of 53BP1 inhibited the radiosensitivity of the HCT116 cell line. Furthermore, the effects of decreased expression of 53BP1 on proliferation and apoptosis were investigated, which revealed that the exposure to radiation doses of 0,2 and 4 Gy remarkably induced tumor proliferation and inhibited tumor cell apoptosis. Then, the effect on the cell cycle was investigated using flow cytometry, which showed that the decreased expression of 53BP1 remarkably increased the percentage of the S-phase in HCT116 cells; the two exposure groups (irradiation of 2 and $4 \mathrm{~Gy}$ ) had higher percentages of S-phase compared with the control groups, although the difference between these two groups was not significant. The previous studies also showed that the decreased expression of 53BP1 can increase the percentage of S-phase (28), which is correlated with cell proliferation $(29,30)$. The results indicated that the decreased expression of 53BP1 prominently enhanced tumor proliferation, inhibited apoptosis, and ushered cells into the proliferation stage, which was associated with radioresistance.

However, the potential mechanisms of 53BP1 impacting the radiosensitivity of $\mathrm{CRC}$ is still uncertain. Han et al (27) found that the increased expression of 53BP1 can affect the expression of certain proteins in the DDR pathway to inhibit DDR, which was related to an enhanced sensitivity to therapy. Recent studies have recognized that ATM-CHK2-p53 as the primary pathway related to apoptosis is involved in the process of DDR repair, and the deletion of critical elements in this pathway results in the resistance to radiotherapy (17). 53BP1 has been found to be critically related to this pathway in the DDR process, thus, this study speculated that 53BP1 participates in regulating apoptosis and cell cycle distribution probably via affecting the ATM-CHK2-p53 pathway and then influencing the response of tumor tissue to radiotherapy. Therefore, after the expression of 53BP1 was decreased in HCT116 cells, mediated by shRNA interference, the proteins and their phosphorylated variants in the ATM-CHK2-p53 pathway were detected by western blot analysis, along with apoptosis-related protein caspase-9, caspase-3, Bax and $\mathrm{Bcl}-2$. The results revealed that the decrease in the expression of 53BP1 decreased the expression of proteins related to the ATM-CHK2-p53 pathway (ATM and CHK2, as well as their phosphorylated products); decreased the expression of apoptosis-related proteins caspase-9, caspase-3 and Bax; and increased the expression of the anti-apoptosis protein Bcl-2. This indicated that 53BP1 affected the response of CRC to radiotherapy through impacting the ATM-CHK2-p53 pathway and that 53BP1 played an important role in affecting the response of tumor cells to radiotherapy. The tumor-bearing nude mouse model was then established to investigate the effects of 53BP1 silencing on radiotherapy in vivo, which showed that 53BP1 silencing notably induced tumor proliferation and that the tumor had a relatively high-proliferation rate even after radiotherapy. Therefore, 53BP1 silencing was verified in vivo to inhibit the efficacy of radiotherapy.

Oncotherapy has now entered the era of precise treatment. Precise radiotherapy is required to maximize efficacy and minimize toxicity. However, no clear factors have been specified to predict the efficacy of radiotherapy. The in vitro and in vivo studies showed that 53BP1 deletion inhibited the ATM-CHK2-p53 pathway for inducing cell proliferation, inhibiting apoptosis, and then inhibiting the radiosensitivity of intestinal cancer. These results provide new perspectives for future studies in this field, but further clinical studies remain to be performed.

\section{References}

1. Shin SJ, Yoon HI, Kim NK, Lee KY, Min BS, Ahn JB, Keum KC and Koom WS: Upfront systemic chemotherapy and preoperative short-course radiotherapy with delayed surgery for locally advanced rectal cancer with distant metastases. Radiat Oncol 6: 99, 2011.

2. Zhang T, Liang ZW, Han J, Bi JP, Yang ZY and Ma H: Double-arc volumetric modulated therapy improves dose distribution compared to static gantry IMRT and 3D conformal radiotherapy for adjuvant therapy of gastric cancer. Radiat Oncol 10: 114, 2015.

3. Wang M, Kern AM, Hülskötter M, Greninger P, Singh A, Pan Y, Chowdhury D, Krause M, Baumann M, Benes CH, et al: EGFR-mediated chromatin condensation protects KRASmutant cancer cells against ionizing radiation. Cancer Res 74: 2825-2834, 2014.

4. Di Micco R, Fumagalli M, Cicalese A, Piccinin S, Gasparini P, Luise C, Schurra C, Garre' M, Nuciforo PG, Bensimon A, et al: Oncogene-induced senescence is a DNA damage response triggered by DNA hyper-replication. Nature 444: 638-642, 2006.

5. Bartkova J, Rezaei N, Liontos M, Karakaidos P, Kletsas D, Issaeva N, Vassiliou LV, Kolettas E, Niforou K, Zoumpourlis VC, et al: Oncogene-induced senescence is part of the tumorigenesis barrier imposed by DNA damage checkpoints. Nature 444: 633-637, 2006

6. Carr SM, Munro S, Zalmas LP, Fedorov O, Johansson C, Krojer T, Sagum CA, Bedford MT, Oppermann U and La Thangue NB: Lysine methylation-dependent binding of 53BP1 to the $\mathrm{pRb}$ tumor suppressor. Proc Natl Acad Sci USA 111: 11341-11346, 2014.

7. Bouwman P, Aly A, Escandell JM, Pieterse M, Bartkova J, van der Gulden H, Hiddingh S, Thanasoula M, Kulkarni A, Yang Q, et al: 53BP1 loss rescues BRCA1 deficiency and is associated with triple-negative and BRCA-mutated breast cancers. Nat Struct Mol Biol 17: 688-695, 2010.

8. Nuciforo PG, Luise C, Capra M, Pelosi G and d'Adda di Fagagna F: Complex engagement of DNA damage response pathways in human cancer and in lung tumor progression. Carcinogenesis 28 : 2082-2088, 2007.

9. Neboori HJ, Haffty BG, Wu H, Yang Q, Aly A, Goyal S, Schiff D, Moran MS, Golhar R, Chen C, et al: Low p53 binding protein 1 (53BP1) expression is associated with increased local recurrence in breast cancer patients treated with breast-conserving surgery and radiotherapy. Int J Radiat Oncol Biol Phys 83: e677-e683, 2012.

10. Squatrito M, Vanoli F, Schultz N, Jasin M and Holland EC: 53BP1 is a haploinsufficient tumor suppressor and protects cells from radiation response in glioma. Cancer Res 72: 5250-5260, 2012. 
11. Ma H, Bi J, Liu T, Ke Y, Zhang S and Zhang T: Icotinib hydrochloride enhances the effect of radiotherapy by affecting DNA repair in colorectal cancer cells. Oncol Rep 33: 1161-1170, 2015.

12. Shi Y, Felley-Bosco E, Marti TM, Orlowski K, Pruschy M and Stahel RA: Starvation-induced activation of ATM/Chk2/p53 signaling sensitizes cancer cells to cisplatin. BMC Cancer 12 : $571,2012$.

13. Chung YM, Park SH, Tsai WB, Wang SY, Ikeda MA, Berek JS, Chen DJ and Hu MC: FOXO3 signalling links ATM to the p53 apoptotic pathway following DNA damage. Nat Commun 3 1000,2012

14. Bartkova J, Horejsí Z, Sehested M, Nesland JM, Rajpert-De Meyts E, Skakkebaek NE, Stucki M, Jackson S, Lukas J and Bartek J: DNA damage response mediators MDC1 and 53BP1: Constitutive activation and aberrant loss in breast and lung cancer, but not in testicular germ cell tumours. Oncogene 26: 7414-7422, 2007.

15. Morales JC, Franco S, Murphy MM, Bassing CH, Mills KD, Adams MM, Walsh NC, Manis JP, Rassidakis GZ, Alt FW, et al: 53BP1 and p53 synergize to suppress genomic instability and lymphomagenesis. Proc Natl Acad Sci USA 103: 3310-3315, 2006.

16. Ausborn NL, Wang T, Wentz SC, Washington MK, Merchant NB, Zhao Z, Shyr Y, Chakravarthy AB and Xia F: 53BP1 expression is a modifier of the prognostic value of lymph node ratio and CA 19-9 in pancreatic adenocarcinoma. BMC Cancer 13: 155, 2013.

17. Squatrito M, Brennan CW, Helmy K, Huse JT, Petrini JH and Holland EC: Loss of ATM/Chk2/p53 pathway components accelerates tumor development and contributes to radiation resistance in gliomas. Cancer Cell 18: 619-629, 2010.

18. Ward IM, Difilippantonio S, Minn K, Mueller MD, Molina JR, Yu X, Frisk CS, Ried T, Nussenzweig A and Chen J: 53BP1 cooperates with p53 and functions as a haploinsufficient tumor suppressor in mice. Mol Cell Biol 25: 10079-10086, 2005.

19. Gorgoulis VG, Vassiliou LV, Karakaidos P, Zacharatos P, Kotsinas A,Liloglou T, Venere M,Ditullio RA Jr, Kastrinakis NG Levy B, et al: Activation of the DNA damage checkpoint and genomic instability in human precancerous lesions. Nature 434: 907-913, 2005
20. Khanna A: DNA damage in cancer therapeutics: A boon or a curse? Cancer Res 75: 2133-2138, 2015.

21. Karanika S, Karantanos T, Li L, Corn PG and Thompson TC: DNA damage response and prostate cancer: Defects, regulation and therapeutic implications. Oncogene 34: 2815-2822, 2015.

22. Tian H, Gao Z, Li H, Zhang B, Wang G, Zhang Q, Pei D and Zheng J: DNA damage response - a double-edged sword in cancer prevention and cancer therapy. Cancer Lett 358: 8-16, 2015.

23. Lai TC, Chow KC, Lin TY, Chiang IP, Fang HY, Chen CY and Ho SP: Expression of 53BP1 as a cisplatin-resistant marker in patients with lung adenocarcinomas. Oncol Rep 24: 321-328, 2010.

24. Li X, Kong X, Kong X, Wang Y, Yan S and Yang Q: 53BP1 sensitizes breast cancer cells to 5-fluorouracil. PLoS One 8: e74928, 2013.

25. Clarke AR, Jones N, Pryde F, Adachi Y and Sansom OJ: 53BP1 deficiency in intestinal enterocytes does not alter the immediate response to ionizing radiation, but leads to increased nuclear area consistent with polyploidy. Oncogene 26: 6349-6355, 2007.

26. Cao L, Xu X, Bunting SF, Liu J, Wang RH, Cao LL, Wu JJ, Peng TN, Chen J, Nussenzweig A, et al: A selective requirement for 53BP1 in the biological response to genomic instability induced by Brcal deficiency. Mol Cell 35: 534-541, 2009.

27. Han X, Zhang L, Chung J, Mayca Pozo F, Tran A, Seachrist DD, Jacobberger JW, Keri RA, Gilmore $\mathrm{H}$ and Zhang Y: UbcH7 regulates 53BP1 stability and DSB repair. Proc Natl Acad Sci USA 111: 17456-17461, 2014

28. Bi J, Huang A, Liu T, Zhang T and Ma H: Expression of DNA damage checkpoint 53BP1 is correlated with prognosis, cell proliferation and apoptosis in colorectal cancer. Int J Clin Exp Pathol 8: 6070-6082, 2015.

29. Sun CC, Chiu HT, Lin YF, Lee KY and Pang JH: Y-27632, a ROCK inhibitor, promoted limbal epithelial cell proliferation and corneal wound healing. PLoS One 10: e0144571, 2015.

30. Li T, Shi HY, Hua YX, Gao C, Xia Q, Yang G and Li B: Effects of allicin on the proliferation and cell cycle of chondrocytes. Int J Clin Exp Pathol 8: 12525-12532, 2015 